
\title{
25 Research Square \\ The Feasibility of the Braden Scale for Assessing Pneumonia After Spontaneous Intracerebral Hemorrhage
}

\section{Yunlong Ding}

Jingjiang People's Hospital

Zhanyi Ji

Zhoukou Central Hospital

Yingmin Zhao

Jingjiang People's Hospital

Chunyan Wu

Jingjiang People's Hospital

Wei Zhang

Jingjiang People's Hospital

\section{Ping Tang}

Jingjiang People's Hospital

Jie Liu

Jingjiang People's Hospital

Zhiqun Gu

Jingjiang People's Hospital

Yan Liu

Jingjiang People's Hospital

Jiali Niu ( $\square$ jialiniu123@sina.com )

Jingjiang People's hospital https://orcid.org/0000-0002-4993-2488

\section{Research article}

Keywords: the Braden scale, intracerebral hemorrhage, pneumonia

Posted Date: July 30th, 2020

DOI: https://doi.org/10.21203/rs.3.rs-47837/v1

License: (c) (1) This work is licensed under a Creative Commons Attribution 4.0 International License.

Read Full License 


\section{Abstract \\ Background}

Stroke-associated pneumonia (SAP) is an infection that commonly occurs in patients with spontaneous intracerebral hemorrhage $(\mathrm{ICH})$ and causes serious burdens. The six subscales of the Braden scale seem to be related to the occurrence of pneumonia. We aimed to evaluate the feasibility of the Braden scale for predicting SAP after spontaneous $\mathrm{ICH}$.

\section{Methods}

Patients with spontaneous ICH who were admitted to Jingjiang People's Hospital and Zhoukou Central Hospital were retrospectively included and divided into two groups: the pneumonia and no pneumonia groups. The Braden scale and ICH-APS-A scale scores and demographic and clinical characteristics were collected, and the differences between the two groups were compared with statistical analyses. Receiver operating characteristic (ROC) curve analysis was used to assess the predictive validity of the Braden scale for SAP after ICH.

\section{Results}

A total of 629 patients with ICH were included in this study, 150 (23.8\%) of whom developed SAP. There were significant differences in age and fasting blood glucose level between the two groups, and the ICHASP-A score in the pneumonia group was significantly higher than that in the no pneumonia group (8.5 \pm 3.9 vs $4.9 \pm 3.2, P<0.01)$. The mean score on the Braden scale in the pneumonia group was significantly lower than that in the no pneumonia group $(16.5 \pm 2.6, P<0.01)$. The area under the curve (AUC) for the ICH-ASP-A score for the prediction of pneumonia after spontaneous ICH was $0.755(95 \% \mathrm{Cl}=0.712-$ 0.798). When the cutoff point was 8 points, the sensitivity was $56.7 \%$ and the specificity was $77.5 \%$. The AUC for the Braden scale for the prediction of pneumonia after ICH was $0.760(95 \% \mathrm{Cl}=0.717-0.804)$. When the cutoff point was 15 points, the sensitivity was $74.3 \%$ and the specificity was $64.7 \%$.

\section{Conclusions}

The Braden scale is effective in predicting pneumonia after ICH. The Braden scale, with a cutoff point of 15 points, is a valid clinical grading scale for predicting SAP after spontaneous $\mathrm{ICH}$.

\section{Background}

Nosocomial infections are complications that frequently occur in patients with spontaneous intracerebral hemorrhage (ICH) [1, 2]. Stroke-associated pneumonia (SAP) accounts for $18 \%$ of all nosocomial infections and is the most common infection in patients with $\mathrm{ICH}$, especially for the elderly[3]. SAP not 
only increases the length of hospital stay and hospital costs $[4,5]$, but is also an important risk factor for poor outcomes after acute stroke[6, 7]. Therefore, it is important to find a scale that is effective in predicting SAP and can help clinicians take early preventative measures to reduce the incidence of SAP[8, 9]. Based on the confirmed risk factors for SAP, including an older age, the male sex, dysphagia, preadmission dependency, stroke severity, etc.[10], the ICH-APS-A scale was established and has been indicated to be effective in predicting the incidence of SAP in patients with ICH [11]. In clinical use of this scale, we found that the Braden scale might be related to pneumonia after spontaneous $\mathrm{ICH}$.

The Braden scale is used to assess the risk of pressure ulcers $[12,13]$, and our prior study indicates that the Braden scale is a valid scale for predicting pneumonia after acute ischemic stroke (AIS)[14]. The scale uses six different risk factors: sensory perception, the ability to respond meaningfully to pressure-related discomfort; skin moisture, the degree to which skin is exposed to moisture; activity, the degree of physical activity; mobility, the ability to change and control one's body position; nutrition, the usual food intake pattern; and friction and shear forces[15]. These indexes included in the Braden scale seem to be related to the occurrence of pneumonia. In this study, we used ICH-APS-A scale as a reference to evaluate the validity of the Braden scale in predicting pneumonia after spontaneous $\mathrm{ICH}$.

\section{Methods}

\section{Study participants}

We retrospectively included consecutive patients with spontaneous $\mathrm{ICH}$ who were admitted to Jingjiang People's Hospital and Zhoukou Central Hospital between January 2015 and August 2018. These two hospitals are the largest tertiary hospitals in the region and are responsible for the treatment of critical illnesses in the area. This study retrospectively included ICH patients admitted to the neurology department who did not undergo surgery. The inclusion criteria were patients who 1 ) were aged $\geq 18$ years; 2 ) were hospitalized with the primary diagnosis of spontaneous ICH according to the World Health Organization criteria[16]; 3) were confirmed to have ICH by head computed tomography; and 4) did not undergo any surgical procedures to treat or reduce the hematoma, including but not limited to minimally invasive hematoma aspiration and craniotomy hematoma removal. The exclusion criteria were patients who acquired pneumonia before admission and patients with primary intraventricular hemorrhage.

\section{Data collection and variable definitions}

Each center selected two senior neurologic physicians to collect the information on the included cases. Cases with discrepancies in the data were evaluated by a third senior physician until an agreement was reached. We collected the patients' demographic and clinical characteristics upon admission, including demographic data (age and sex), risk factors (history of smoking and drinking, history of chronic obstructive pulmonary disease (COPD), hypertension, hyperlipidemia, diabetes, coronary heart disease, atrial fibrillation (AF), heart failure (HF), peripheral vascular disease (PVD) and history of stroke or transient ischemic attacks (TIAs)), laboratory examination results (diastolic blood pressure and systolic blood pressure), the international normalized ratio (INR), serum creatinine levels, fasting blood glucose 
levels, total cholesterol levels, triglyceride levels, low-density lipoprotein cholesterol levels, high-density lipoprotein cholesterol levels and glycosylated hemoglobin levels, and the ICH-ASP-A scale and Braden scale scores at admission.

ICH-ASP-A scale was measured at admission, including items on age, current smoking status, excess alcohol consumption, COPD, prestroke level of dependence (modified Rankin Scale (mRS $\geq 3$ )), Glasgow coma scale (GCS) score at admission, National Institutes of Health Stroke Scale (NIHSS) score at admission, dysphagia, the infratentorial location and extent of ventricle involvement (Table 1[11]). The total score ranges from $0-23$, with a lower score being associated with a lower risk.

Nurses administered the Braden scale at $24 \mathrm{~h}$ after admission, which is composed of six subscales: sensory perception, skin moisture, activity, mobility, nutrition, friction and shear forces. The score for friction and shear forces ranges from 1 (worst) to 3 (best), and the other scores range from 1 to 4 . The sum of the scores ranges from 6 to 23 (Table 2 [17]).

Pneumonia after $\mathrm{ICH}$ was diagnosed according to the Centers for Disease Control and Prevention criteria[18] for hospital-acquired pneumonia on the basis of clinical and laboratory indexes of respiratory tract infection (fever, productive cough with purulent sputum, auscultatory respiratory crackles, bronchial breathing, or positive sputum culture) and supported by abnormal chest radiographic findings.

\section{Statistical analysis}

Statistical differences between the pneumonia and no pneumonia groups were performed using SPSS version 21.0 (SPSS Inc., Chicago, IL, USA). Student's t test was used for normally distributed variables (described as the mean $\pm S D$ ), the Mann-Whitney $U$ test was used for nonnormally distributed continuous variables, and Fisher's exact test or the chi-square test was used for dichotomous variables. A $P$-value of $<0.05$ was considered statistically significant. Then, receiver operating characteristic (ROC) curve analysis was performed to investigate the predictive validity of the Braden scale for pneumonia after ICH. An area under the curve (AUC) of 0.97-1.00 indicates excellent accuracy; 0.93 to 0.96 indicates very good accuracy; and 0.75 to 0.92 indicates good accuracy. However, an AUC of $<0.75$ indicates obvious deficiencies, and an AUC of 0.5 indicates that the test has no predictive ability[19].

\section{Results}

Eight hundred and eighteen patients with with spontaneous ICH were admitted to Jingjiang People's Hospital and Zhoukou Central Hospital between January 2015 and August 2018. Among them, 3 patients acquired pneumonia before admission, 80 patients underwent surgery, 48 patients had incomplete or missing data, and 58 patients were discharged from the hospital during hospitalization. Ultimately, 629 patients with spontaneous ICH were retrospectively included in this study; 150 of the 629 (23.8\%) patients were included in the hospital-acquired pneumonia group, and 479 (76.2\%) were included in the no pneumonia group. There were 380 males $(60.4 \%)$ and 249 females (39.6\%), and their mean age was $66.1 \pm 13.4$ years. 


\section{Demographic and clinical characteristics}

The demographic data (sex), risk factors (history of smoking and drinking, history of COPD, hypertension, hyperlipidemia, diabetes, coronary heart disease, AF, HF, PVD and past stroke or TIA), and laboratory examination results (diastolic blood pressure and systolic blood pressure, INR, serum creatinine levels, and total cholesterol, triglyceride, low-density lipoprotein cholesterol, high-density lipoprotein cholesterol and glycosylated hemoglobin levels) showed no significant differences between the pneumonia and no pneumonia groups. There were significant differences in age and the fasting blood glucose level between the two groups. A significant difference was also observed between the two groups in the ICH-ASP-A score, which was significantly higher in the pneumonia group than in the no pneumonia group $(8.5 \pm 3.9 \mathrm{vs}$ 4.9 $\pm 3.2, P<0.01)$ (Table 3).

The mean score on the Braden scale in the pneumonia group was $14.1 \pm 2.4$, which was significantly lower than that in the no pneumonia group $(16.5 \pm 2.6, P<0.01)$. (Table 1). All six subscale scores on the Braden scale significantly differed between the two groups (Table 4).

\section{The validity of the association between the Braden scale/ICH-ASP-A score and pneumonia after spontaneous ICH}

The AUC for the Braden scale for the prediction of pneumonia after spontaneous $\mathrm{ICH}$ was $0.760(95 \% \mathrm{Cl}=$ $0.717-0.804)$. When the cutoff point was 15 points, the sensitivity was $74.3 \%$ and the specificity was $64.7 \%$ (Fig. 1).

The AUC for the ICH-ASP-A score for the prediction of pneumonia after spontaneous intracerebral hemorrhage was $0.755(95 \% \mathrm{Cl}=0.712-0.798)$. When the cutoff point was 8 points, the sensitivity was $56.7 \%$ and the specificity was $77.5 \%$ (Fig. 2 ).

\section{Discussion}

In this study, we evaluated the correlation between the Braden scale and ASP after ICH. We found that patients with ICH who had a lower Braden scale score were more likely to develop SAP. The AUC for the Braden scale for the prediction of pneumonia after spontaneous $\mathrm{ICH}$ was $0.760(95 \% \mathrm{Cl}=0.717-0.804)$. When the cutoff point was 15 points, the sensitivity was $74.3 \%$ and the specificity was $64.7 \%$. Moreover, we verified the correlation between the ICH-ASP-A score and the occurrence of SAP after ICH in these patients and inferred that the Braden scale was not inferior to the ICH-ASP-A score in predicting the incidence of SAP in ICH patients. ICH-ASP-A score is evaluated by professional doctors, and the Braden scale can be quickly assessed by nurses, which also provides a new way to predict pneumonia after ICH.

Our previous study verified the correlation between the Braden scale and SAP after AIS. The AUC for the Braden scale for the prediction of pneumonia after AIS was $0.883(95 \% \mathrm{Cl}=0.828-0.937)$. When the cutoff point was 18 points, the sensitivity was $83.2 \%$ and the specificity was $84.2 \%$ [14]. This result suggests that the efficacy of the Braden scale in predicting pneumonia after $\mathrm{ICH}$ is lower than that in 
predicting pneumonia after AIS. After we compared the data, we found that the average Braden scale score of all the $\mathrm{ICH}$ patients in this study was $15.91 \pm 2.77$, which was lower than that reported in the previous study of AIS patients (18.96 \pm 2.71$)$ and suggests that compared with AIS patients, ICH patients may have poorer mobility and a poorer nutritional status at admission. Therefore, we speculate that ICH patients may have more severe nerve functional impairment at admission, decreasing the sensitivity of the Braden scale in predicting pneumonia after $\mathrm{ICH}$. However, the sensitivity was $74.3 \%$ with a cutoff point of 15 points, which still suggests that it is feasible for predicting SAP after $\mathrm{ICH}$.

Risk factors for SAP after stroke include the following [20-22]: age, sex, the NIHSS score, dysphagia, current smoking status, the GCS score, and dysphagia. The ICH-ASP-A is a scale that combines these risk factors[11]. Our research suggests that the ICH-ASP-A is effective in predicting SAP after ICH and clarifies the correlation between the Braden scale and SAP after ICH. Although the Braden scale does not include these risk factors, the indexes in the Braden scale are associated with some risk factors for SAP. The nutritional indicators are related to the patient's age and dysphagia. Sensory perception and mobility are related to the NIHSS score. Skin moisture and activity are related to the patient's GCS score. Given that the sensitivity of the Braden scale is not weaker than that of the ICH-ASP-A, additional studies based on age, dysphagia, the NIHSS score, and the GCS score should be conducted to assess the feasibility of predicting pneumonia after $\mathrm{ICH}$.

Patients with ICH experience different neurological deficits and levels of consciousness at different times. Therefore, the Braden score, NIHSS score, and GCS score assessed at different time points also differ. The ICH-ASP-A is administered to patients at admission[11]. We administered the Braden scale at 24 hours after admission to evaluate the incidence of SAP after AIS, and we confirmed that the 24-hour Braden score can effectively predict poststroke pneumonia. In this study, we also administered the Braden scale to patients at 24 hours after admission because cerebral hemorrhage is more likely to progress within 24 hours after onset, most patients' conditions tend to stabilize after 24 hours of onset, and the possibility of increased bleeding is relatively small. Therefore, the assessments of neurological deficit performed at 24 hours are more indicative of the progression of patients' conditions than those performed at admission.

Our study did not include patients with $\mathrm{ICH}$ who underwent surgery because the purpose of this study was to evaluate the sensitivity of the Braden scale in predicting poststroke pneumonia; the eventual goal was to screen high-risk patients and take effective preventive measures, thereby improving their prognosis. ICH patients undergoing surgery are already at high risk of lung infection due to anesthesia and tracheal intubation[23-25]. These patients need medical staff to take necessary measures to prevent pneumonia after stroke. In addition, there is no correlation between the risk factors for the need for these operations and the Braden scale, but the presence of correlations may increase the incidence of pneumonia and affect the sensitivity of the study. Therefore, we excluded all patients who underwent surgical treatment. 
Our study has some limitations. First, our study included only hospitalized patients, and the patients who were treated in outpatient clinics or died in the emergency department were not included. Second, we cannot rule out the possibility that unmeasured confounders might have some impact on the risk of SAP after ICH. Finally, we recorded the Braden score at 24 hours after admission, but we are not sure if this time point is the best.

\section{Conclusions}

In summary, the Braden scale is effective in predicting pneumonia after $\mathrm{ICH}$. With a cutoff point of 15 points, the Braden scale is a valid clinical grading scale for predicting SAP after spontaneous ICH.

\section{Abbreviations}

$\mathrm{ICH}$

Intracerebral Hemorrhage; SAP:Stroke-Associated Pneumonia; AIS:Acute Ischemic Stroke; COPD:Chronic Obstructive Pulmonary Disease; AF:Atrial Fibrillation; HF:Heart Failure; PVD:Peripheral Vascular Disease; TIAs:Transient Ischemic Attacks; INR:International Normalized Ratio; mRS:modified Rankin Scale; GCS:Glasgow Coma Scale; NIHSS:National Institutes of Health Stroke Scale; ROC:Receiver Operating Characteristic; AUC:Area Under the Curve.

\section{Declarations}

\section{Ethics approval and consent to participate}

The study was approved by the medical ethics committee of Jingjiang People's Hospital and Zhoukou Central Hospital. Because it was a retrospective study and did not include any personal information related to the participants, the need to obtain written informed consent was waived. The treatment of each participant during hospitalization was approved by the patient or their close family member, and written informed consent form was obtained before treatment.

\section{Consent for publication}

Not applicable.

\section{Availability of data and materials}

The datasets analysed during the current study are available from the corresponding author on reasonable request.

\section{Conflict of interest}

The authors declare that they have no conflicts of interest. 


\section{Funding}

This work was funded by the Fifth "311 Project" Scientific Research Funding Project in Taizhou (RCPY202004). The funder had no role in the study design, data collection, data analysis, data interpretation, writing of the report, decision to publish, or preparation of the manuscript.

\section{Authors' contributions}

Study concept and design: JLN, YL, YLD, ZYJ and YMZ. Data analysis: YLD, JLN, CYW and WZ. Data collection: YLD, ZYJ, JLN, WZ, PT, JL, ZQG and YL. Writing of the manuscript: YLD, ZYJ, JLN, YMZ, CYW, WZ, PT, ZQG and JL. Revising the manuscript: YL and JLN. Final approval of the manuscript: all.

\section{Acknowledgements}

None.

\section{References}

1. Murthy SB, Moradiya Y, Shah J, Merkler AE, Mangat HS, ladacola C, et al. Nosocomial Infections and Outcomes after Intracerebral Hemorrhage: A Population-Based Study. Neurocrit Care. 2016;25(2):178-84.

2. Lindner A, Kofler M, Rass V, lanosi B, Gaasch M, Schiefecker AJ, et al. Early Predictors for Infectious Complications in Patients With Spontaneous Intracerebral Hemorrhage and Their Impact on Outcome. Front Neurol. 2019;10:817.

3. Hinduja A, Dibu J, Achi E, Patel A, Samant R, Yaghi S. Nosocomial infections in patients with spontaneous intracerebral hemorrhage. Am J Crit Care. 2015;24(3):227-31.

4. Ali AN, Howe J, Majid A, Redgrave J, Pownall S, Abdelhafiz AH. The economic cost of strokeassociated pneumonia in a UK setting. Top Stroke Rehabil. 2018;25(3):214-23.

5. Ingeman A, Andersen G, Hundborg HH, Svendsen ML, Johnsen SP. In-hospital medical complications, length of stay, and mortality among stroke unit patients. Stroke. 2011;42(11):3214-8.

6. Teh WH, Smith CJ, Barlas RS, Wood AD, Bettencourt-Silva JH, Clark AB, et al. Impact of strokeassociated pneumonia on mortality, length of hospitalization, and functional outcome. Acta Neurol Scand. 2018;138(4):293-300.

7. Westendorp WF, Nederkoorn PJ, Vermeij JD, Dijkgraaf MG, van de Beek D. Post-stroke infection: a systematic review and meta-analysis. BMC Neurol. 2011;11:110.

8. Li X, Wu M, Sun C, Wang ZZ, Zheng F. X, et al. Using machine learning to predict stroke-associated pneumonia in Chinese patients with acute ischemic stroke. Eur J Neurol. 2020. 
9. Palli C, Fandler S, Doppelhofer K, Niederkorn K, Enzinger C, Vetta C, et al. Early Dysphagia Screening by Trained Nurses Reduces Pneumonia Rate in Stroke Patients: A Clinical Intervention Study. Stroke. 2017:48(9):2583-85.

10. Finlayson O, Kapral M, Hall R, Asllani E, Selchen D, Saposnik G. Risk factors, inpatient care, and outcomes of pneumonia after ischemic stroke. Neurology. 2011;77(14):1338-45.

11. Ji R, Shen H, Pan Y, Du W, Wang P, Liu G, et al. Risk score to predict hospital-acquired pneumonia after spontaneous intracerebral hemorrhage. Stroke. 2014;45(9):2620-8.

12. Wei M, Wu L, Chen Y, Fu Q, Chen W, Yang D. Predictive Validity of the Braden Scale for Pressure Ulcer Risk in Critical Care: A Meta-Analysis. Nurs Crit Care. 2020;25(3):165-70.

13. Watkins AA, Castillo-Angeles M, Calvillo-Ortiz R, Guetter CR, Eskander MF, Ghaffarpasand E, et al. Braden scale for pressure ulcer risk predicts rehabilitation placement after pancreatic resection. HPB (Oxford). 2019;21(7):923-27.

14. Ding Y, Yan Y, Niu J, Zhang Y, Gu Z, Tang P, et al. Braden scale for assessing pneumonia after acute ischaemic stroke. BMC Geriatr. 2019;19(1):259.

15. Griswold LH, Griffin RL, Swain T, Kerby JD. Validity of the Braden Scale in grading pressure ulcers in trauma and burn patients. J Surg Res. 2017;219:151-57.

16. Stroke-1989. Recommendations on stroke prevention, diagnosis, and therapy. Report of the WHO Task Force on Stroke and other Cerebrovascular Disorders. Stroke. 1989;20(10):1407-31.

17. Alderden J, Cummins MR, Pepper GA, Whitney JD, Zhang Y, Butcher R, et al. Midrange Braden Subscale Scores Are Associated With Increased Risk for Pressure Injury Development Among Critical Care Patients. J Wound Ostomy Continence Nurs. 2017;44(5):420-28.

18. Garner JS, Jarvis WR, Emori TG, Horan TC, Hughes JM. [CDC definitions for nosocomial infections 1988]. Z Arztl Fortbild (Jena). 1991;85(17):818-27.

19. Swets JA. Measuring the accuracy of diagnostic systems. Science. 1988;240(4857):1285-93.

20. Smith CJ, Bray BD, Hoffman A, Meisel A, Heuschmann PU, Wolfe CD, et al. Can a novel clinical risk score improve pneumonia prediction in acute stroke care? A UK multicenter cohort study. J Am Heart Assoc. 2015;4(1):e001307.

21. Kumar S, Marchina S, Massaro J, Feng W, Lahoti S, Selim M, et al. ACDD(4) score: A simple tool for assessing risk of pneumonia after stroke. J Neurol Sci. 2017;372:399-402.

22. Westendorp WF, Vermeij JD, Hilkens NA, Brouwer MC, Algra A, van der Worp HB, et al. Development and internal validation of a prediction rule for post-stroke infection and post-stroke pneumonia in acute stroke patients. Eur Stroke J. 2018;3(2):136-44.

23. Hilker R, Poetter C, Findeisen N, Sobesky J, Jacobs A, Neveling M, et al. Nosocomial pneumonia after acute stroke: implications for neurological intensive care medicine. Stroke. 2003;34(4):975-81.

24. Alsumrain M, Melillo N, Debari VA, Kirmani J, Moussavi M, Doraiswamy V, et al. Predictors and outcomes of pneumonia in patients with spontaneous intracerebral hemorrhage. J Intensive Care Med. 2013;28(2):118-23. 
25. Lioutas VA, Marchina S, Caplan LR, Selim M, Tarsia J, Catanese L, et al. Endotracheal Intubation and In-Hospital Mortality after Intracerebral Hemorrhage. Cerebrovasc Dis. 2018;45(5-6):270-78.

\section{Tables}

Table 1. Intracerebral hemorrhage-associated pneumonia score without hematoma volume (ICH-APS-A).

\begin{tabular}{|lllllll|}
\hline Items & Score & & & & \\
\cline { 2 - 6 } & 0 & 1 & 2 & 3 & 4 & 5 \\
\hline Age group (year) & $\leq 59$ & - & $60-69$ & $70-79$ & $\geq 80$ & - \\
\hline Current smoking & No & Yes & - & - & - & - \\
\hline Excess alcohol consumption & No & Yes & - & - & - & - \\
\hline COPD & No & - & - & - & - & - \\
\hline Pre-stroke dependence (mRS $\geq 3)$ & No & - & Yes & - & - & - \\
\hline Admission GCS score & 15 & - & $3-14$ & - & - & - \\
\hline Admission NIHSS score & $0-5$ & $6-10$ & $11-15$ & $\geq 16$ & - & - \\
\hline Dysphagia & No & - & Yes & - & - & - \\
\hline Infratentorial location & No & Yes & - & - & - & - \\
\hline Extension into ventricles & No & Yes & - & - & - & - \\
\hline
\end{tabular}

COPD: chronic obstructive pulmonary disease; mRS: modified Rankin Scale; GCS, Glasgow coma scale; and NIHSS, National Institutes of Health Stroke Scale.

Table 2. The Braden Scale. 


\begin{tabular}{|c|c|c|c|c|}
\hline \multirow[t]{2}{*}{ Items } & \multicolumn{4}{|l|}{ Score } \\
\hline & 1 & 2 & 3 & 4 \\
\hline $\begin{array}{l}\text { Sensory } \\
\text { perception }\end{array}$ & Completely limited & Very limited & Slightly limited & No impairment \\
\hline Skin moisture & Constantly moist & Very moist & $\begin{array}{l}\text { Occasionally } \\
\text { moist }\end{array}$ & Rarely moist \\
\hline Activity & Bedfast & Chairfast & $\begin{array}{l}\text { Walks } \\
\text { occasionally }\end{array}$ & $\begin{array}{l}\text { Walks } \\
\text { frequently }\end{array}$ \\
\hline Mobility & $\begin{array}{l}\text { Completely } \\
\text { immobile }\end{array}$ & Very limited & Slightly limited & No limitations \\
\hline Nutrition & Very poor & $\begin{array}{l}\text { Probably } \\
\text { inadequate }\end{array}$ & Adequate & Excellent \\
\hline $\begin{array}{l}\text { Friction and } \\
\text { shear }\end{array}$ & Problem & Potential problem & $\begin{array}{l}\text { No apparent } \\
\text { problem }\end{array}$ & - \\
\hline
\end{tabular}

Table 3. Demographic and clinical characteristics of the patients in the two groups. 


\begin{tabular}{|c|c|c|c|}
\hline & No pneumonia $(n=479)$ & $\begin{array}{l}\text { Pneumonia } \\
(n=150)\end{array}$ & $P$ value \\
\hline \multicolumn{4}{|l|}{ Demographic } \\
\hline Age/year & $64.2 \pm 13.0$ & $72.1 \pm 12.9$ & $<0.01$ \\
\hline Male (case, \%) & $292(61.0)$ & $88(58.7)$ & 0.633 \\
\hline \multicolumn{4}{|l|}{ Risk factors } \\
\hline Smoking status (case, \%) & 152(31.7) & $44(29.3)$ & 0.614 \\
\hline Drinking status (case, \%) & $93(19.4)$ & $28(18.7)$ & 0.906 \\
\hline COPD (case, \%) & $6(1.3)$ & $2(1.3)$ & 1.000 \\
\hline Hypertension (case, \%) & $333(69.5)$ & 107(71.3) & 0.760 \\
\hline Diabetes (case, \%) & 137(28.6) & $51(34.0)$ & 0.221 \\
\hline Hyperlipidemia (case, \%) & $20(4.2)$ & $6(4.0)$ & 1.000 \\
\hline Coronary heart disease (case, \%) & $48(10.0)$ & $19(12.7)$ & 0.365 \\
\hline AF (case, \%) & $50(10.4)$ & $12(8.0)$ & 0.435 \\
\hline HF (case, \%) & $1(0.2)$ & $1(0.7)$ & 0.420 \\
\hline Valvulopathy (case, \%) & $6(1.3)$ & $5(3.3)$ & 0.144 \\
\hline PVD (case, \%) & $2(0.4)$ & $0(0)$ & 1.000 \\
\hline Stroke/TIA (case, \%) & $5(1.0)$ & $0(0)$ & 0.598 \\
\hline \multicolumn{4}{|l|}{ Laboratory examination } \\
\hline $\mathrm{SBP}(\mathrm{mmHg})$ & $144.6 \pm 20.5$ & $146.0 \pm 21.8$ & 0.488 \\
\hline $\mathrm{DBP}(\mathrm{mmHg})$ & $85.5 \pm 13.2$ & $85.2 \pm 13.3$ & 0.817 \\
\hline INR & $1.0 \pm 0.3$ & $1.1 \pm 0.9$ & 0.154 \\
\hline Serum creatinine (umol/L) & $81.8 \pm 58.2$ & $88.7 \pm 32.1$ & 0.167 \\
\hline Fasting blood glucose (mmol/L) & $6.0 \pm 2.2$ & $7.3 \pm 3.2$ & $<0.01$ \\
\hline $\mathrm{TC}(\mathrm{mmol} / \mathrm{L})$ & $4.3 \pm 1.1$ & $4.4 \pm 1.6$ & 0.305 \\
\hline TG (mmol/L) & $1.5 \pm 0.9$ & $1.6 \pm 2.5$ & 0.398 \\
\hline $\mathrm{HDL}(\mathrm{mmol} / \mathrm{L})$ & $1.1 \pm 0.5$ & $1.2 \pm 0.3$ & 0.180 \\
\hline LDL (mmol/L) & $2.7 \pm 1.0$ & $2.7 \pm 1.1$ & 0.834 \\
\hline $\mathrm{HbA1c}(\%)$ & $6.4 \pm 1.5$ & $6.4 \pm 1.4$ & 0.885 \\
\hline
\end{tabular}




\begin{tabular}{|llll|}
\hline Scores & & & \\
\hline ICH-ASP-A & $4.9 \pm 3.2$ & $8.5 \pm 3.9$ & $<0.01$ \\
\hline Braden Scale & $16.5 \pm 2.6$ & $14.1 \pm 2.4$ & $<0.01$ \\
\hline
\end{tabular}

COPD: chronic obstructive pulmonary disease; AF: atrial fibrillation; HF: heart failure; PVD: peripheral vascular disease; TIA, transient ischemic attack; SBP: systolic pressure; DBP: diastolic pressure; INR: international normalized ratio; TC: total cholesterol; TG: triacylglycerol; HDL: high-density lipoprotein cholesterol; LDL: low-density lipoprotein cholesterol; HbA1c: glycosylated hemoglobin; GCS, Glasgow coma scale.

Table 4. The Braden scale scores for the two groups (mean \pm SD)

\begin{tabular}{|llll|}
\hline Braden scale & $\begin{array}{l}\text { No pneumonia } \\
(\mathrm{n}=479)\end{array}$ & $\begin{array}{l}\text { Pneumonia } \\
(\mathrm{n}=150)\end{array}$ & $P$ value \\
\hline Sensory perception & $3.4 \pm 0.7$ & $2.8 \pm 0.7$ & $<0.01$ \\
\hline Skin moisture & $3.7 \pm 0.5$ & $3.5 \pm 0.6$ & $<0.01$ \\
\hline Activity & $1.3 \pm 0.8$ & $1.1 \pm 0.6$ & 0.001 \\
\hline Mobility & $3.0 \pm 0.8$ & $2.4 \pm 0.8$ & $<0.01$ \\
\hline Nutrition & $2.9 \pm 0.4$ & $2.7 \pm 0.6$ & $<0.01$ \\
\hline Friction and shear & $2.1 \pm 0.7$ & $1.7 \pm 0.6$ & $<0.01$ \\
\hline Sum score & $16.5 \pm 2.6$ & $14.1 \pm 2.4$ & $<0.01$ \\
\hline
\end{tabular}

The scores for all six subscales of the Braden scale were significantly different between the two groups.

\section{Figures}




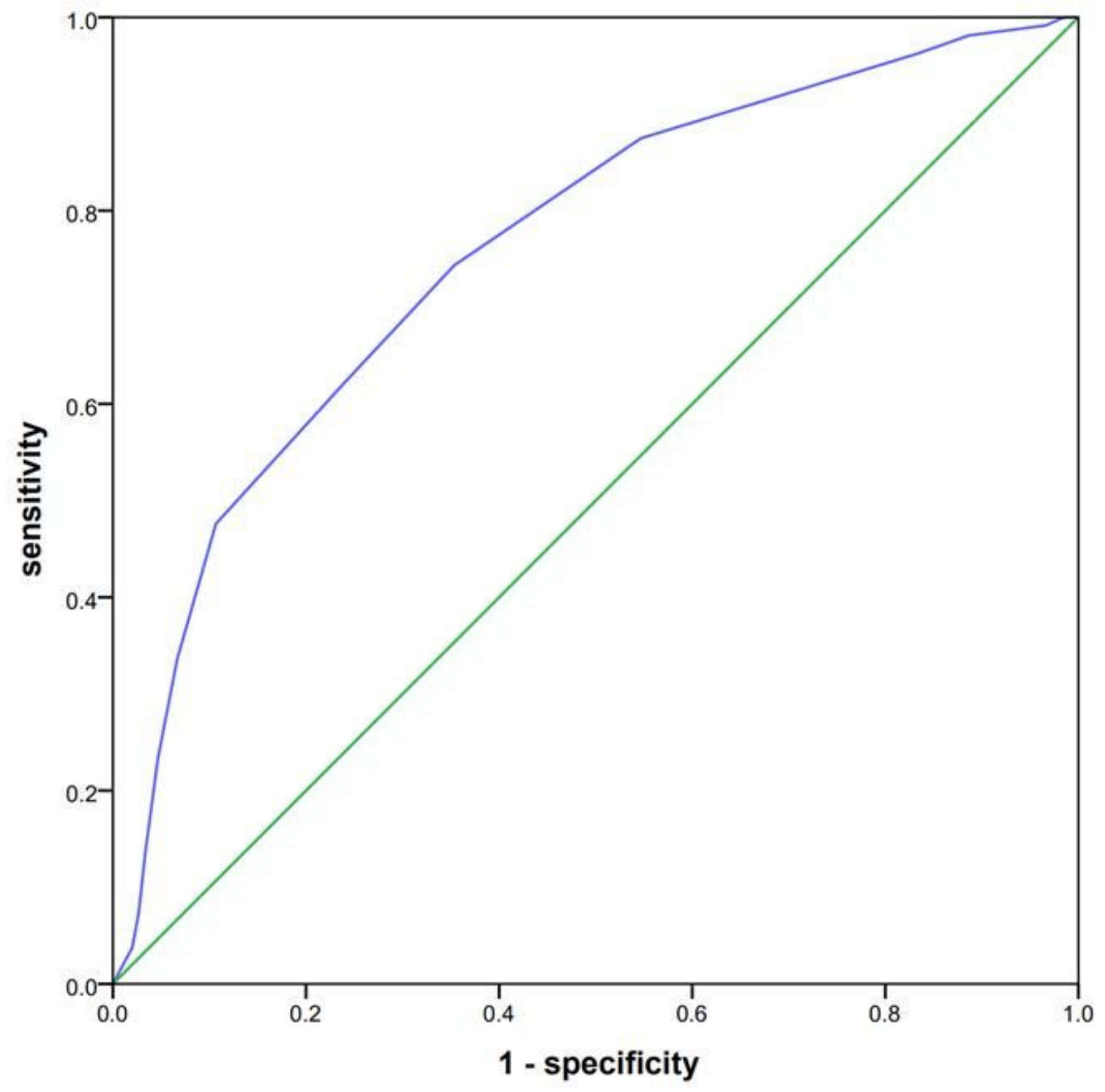

\section{Figure 1}

ROC curve for the Braden scale. The AUC for the Braden scale for the prediction of pneumonia after spontaneous ICH was $0.760(95 \% \mathrm{Cl}=0.717-0.804)$. When the cutoff point was 15 points, the sensitivity was $74.3 \%$ and the specificity was $64.7 \%$. 


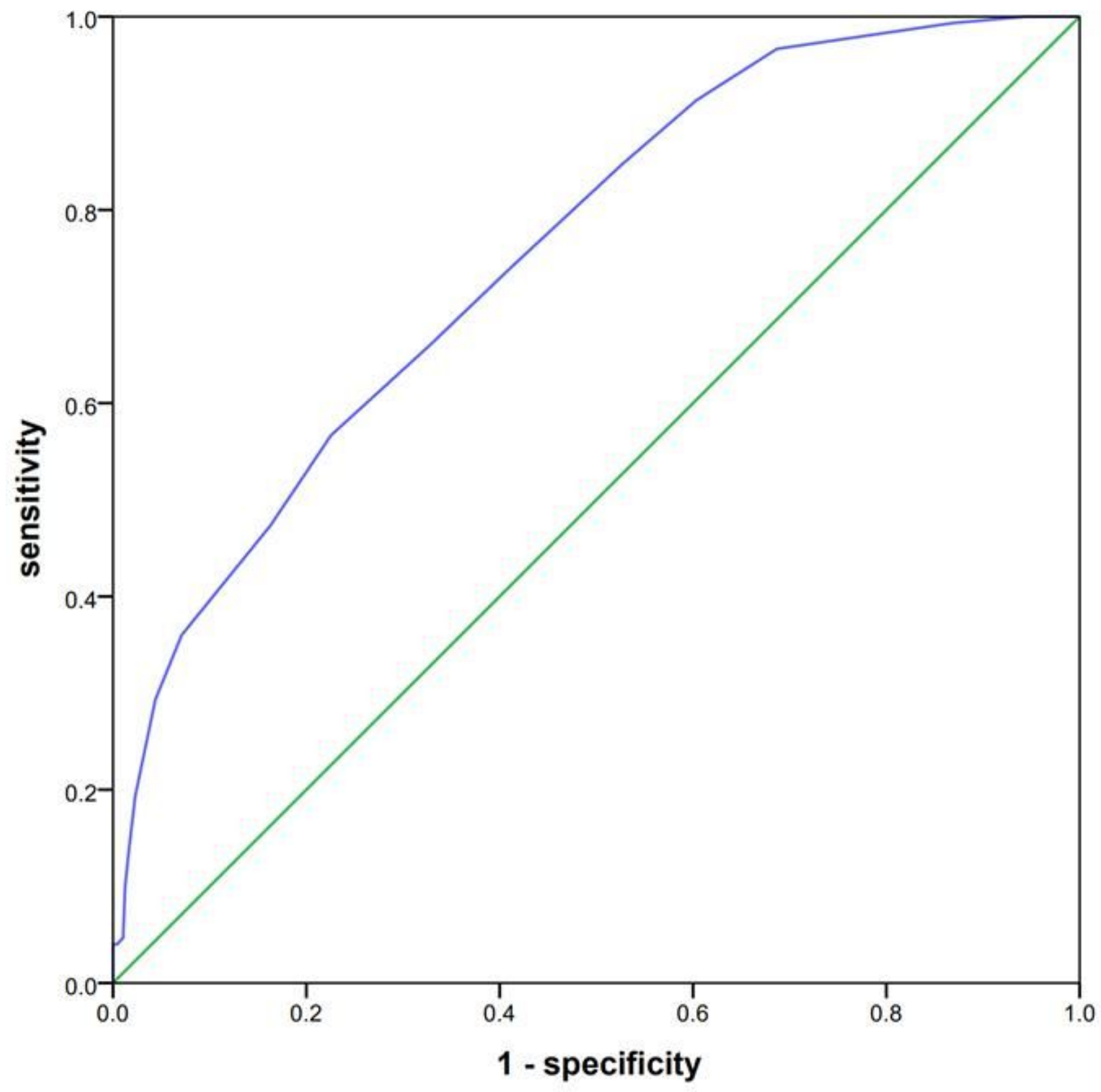

Figure 2

ROC curve for the ICH-ASP-A. The AUC for the ICH-ASP-A score for the prediction of pneumonia after spontaneous intracerebral hemorrhage was $0.755(95 \% \mathrm{Cl}=0.712-0.798)$. When the cutoff point was 8 points, the sensitivity was $56.7 \%$ and the specificity was $77.5 \%$. 